\title{
Ice giant system exploration within ESA's Voyage 2050
}

Leigh N. Fletcher ${ }^{1}$ (D) - Ravit Helled ${ }^{2}$. Elias Roussos ${ }^{3}$. Geraint Jones ${ }^{4}$. Sébastien Charnoz $^{5}$. Nicolas André ${ }^{6}$. David Andrews ${ }^{7}$. Michele Bannister ${ }^{8}$. Emma Bunce ${ }^{1}$. Thibault Cavalié ${ }^{9,10}$. Francesca Ferri ${ }^{11}$. Jonathan Fortney ${ }^{12}$. Davide Grassi ${ }^{13}$. Léa Griton ${ }^{14}$. Paul Hartogh ${ }^{3}$. Ricardo Hueso ${ }^{15}$. Yohai Kaspi ${ }^{16}$ - Laurent Lamy ${ }^{17}$. Adam Masters ${ }^{18} \cdot$ Henrik Melin $^{1}$. Julianne Moses ${ }^{19}$. Oliver Mousis ${ }^{20}$. Nadine Nettleman ${ }^{21}$. Christina Plainaki ${ }^{22}$. Jürgen Schmidt ${ }^{23}$. Amy Simon ${ }^{24}$. Gabriel Tobie ${ }^{25}$. Paolo Tortora ${ }^{26}$. Federico Tosi ${ }^{13} \cdot$ Diego Turrini ${ }^{13}$

Received: 4 September 2020 / Accepted: 6 May 2021 / Published online: 1 July 2021

(C) The Author(s) 2021

\begin{abstract}
Of all the myriad environments in our Solar System, the least explored are the distant Ice Giants Uranus and Neptune, and their diverse satellite and ring systems. These 'intermediate-sized' worlds are the last remaining class of Solar System planet to be characterised by a dedicated robotic mission, and may shape the paradigm for the most common outcome of planetary formation throughout our galaxy. In response to the 2019 European Space Agency call for scientific themes in the 2030s and 2040s (known as Voyage 2050), we advocated that an international partnership mission to explore an Ice Giant should be a cornerstone of ESA's science planning in the coming decade, targeting launch opportunities in the early 2030s. This article summarises the inter-disciplinary science opportunities presented in that White Paper [1], and briefly describes developments since 2019.
\end{abstract}

Keywords Planetary exploration · Ice Giants · Uranus · Neptune

\section{Introduction}

The frozen realm of the Ice Giants, from Uranus (20 AU) to Neptune (30 AU), remains largely unexplored when compared to our comprehensive characterisation of Jupiter (by Galileo, Juno, and the forthcoming Jupiter Icy Moons Explorer and

Leigh N. Fletcher

leigh.fletcher@le.ac.uk

Extended author information available on the last page of the article. 
Europa Clipper) and Saturn (Cassini). Voyager's 'Grand Tour' of the Solar System remains the only mission to provide close-up views of the Uranus and Neptune systems via flybys more than three decades ago. Voyager 2 encountered both worlds within a three-year 'golden era' for Ice Giant science between 1986 and 1989, summarised at the time by Stone \& Miner [2, 3] in special issues of Science, which also featured the first close-up images of both worlds by Smith et al. [4, 5]. In the decades since Voyager's encounters, ground- and space-based astronomy have continued to push the limits of our knowledge of the atmospheres of these intermediate-sized worlds, but there have been no new in situ measurements of their unique magnetospheres and plasma environments; no new measurement constraints on their interior density distributions and thermal evolution; and no new close-up remote sensing of their icy satellites and rings. Indeed, given the illumination conditions in the late 1980s (southern summer on Uranus, southern spring on Neptune), the great distance separating Voyager 2 from its targets, and the brevity inherent in flyby missions, there are vast and mysterious terrains that have never been observed by robotic eyes. And we have never directly sampled the atmospheric composition of an Ice Giant, a key missing piece of the planet-formation puzzle. A mission to an Ice Giant would allow humanity to complete its first detailed reconnaissance of the eight primary planets in our Solar System.

Given the enormous potential for scientific discovery, the past decade has seen a number of mission concepts and scientific reviews, of which the Voyage 2050 White Paper on the Scientific Potential of Orbital Missions to Uranus and Neptune by Fletcher et al. [1] is the most recent. We will not replicate that paper in full, but Section 2 reviews the recent history of Ice Giant mission concepts, Section 3 reiterates the key scientific questions that an Ice Giant System mission could address, and Section 4 summarises the challenges and opportunities presented by a 'cornerstone' mission within ESA's Voyage 2050 programme.

\section{Ice giants in ESA's cosmic vision}

The challenge of combining a multi-year cruise phase with an ambitious Ice Giant orbital tour; a sophisticated interplanetary spacecraft with a broad suite of capable instruments; all operating at enormous distances from the Sun and relying on power generation via radioisotope decay, implies that any future Ice Giant mission must be a broad international partnership. Strategic partnerships were reflected in the proposed medium-class ( $€ 0.5 \mathrm{bn}$ ) Uranus Pathfinder mission concepts submitted to ESA by Arridge et al. in the M3 (2010) and M4 (2014) opportunities [6], and in the largeclass mission themes ( $€ 1$ bn) submitted to ESA in 2013 to explore Uranus [7], Neptune [8], or both worlds [9]. Although these efforts failed to proceed to a formal study phase, ESA's Senior Survey Committee ${ }^{1}$ noted that “...the exploration of the icy giants appears to be a timely milestone, fully appropriate for an L class mission. The whole planetology community would be involved in the various aspects of this

\footnotetext{
${ }^{1} \mathrm{http}: / /$ sci.esa.int/cosmic-vision/53261-report-on-science-themes-for-the-12-and-13-missions/
} 
mission... the SSC recommends that every effort is made to pursue this theme through other means, such as cooperation on missions led by partner agencies."

In the US, the National Academies decadal survey in planetary science 2013$2022^{2}$ listed a Uranus Orbiter and Probe as its third highest priority, after the Mars Perseverance (2020) rover mission and Europa Clipper mission, both of which are now underway. This enthusiasm for Ice Giants from both sides of the Atlantic led to a joint study (2016-17), which looked closely at a number of potential mission architectures [10]. Since then, ESA has considered a palette of potential contributions to a US-led mission ${ }^{3}$ (2018-19), including secondary orbiters and atmospheric probes, as reviewed by Simon et al. [11] and advocated by Mousis et al. ([12], this issue). At the time of our submission to Voyage 2050, it had been hoped that such a contribution, at the $€ 0.5 \mathrm{bn}$ level, might be included in a proposed increase in ESA's science budget in 2019. However, the challenging timescales of the US mission planning process proved problematic. Ice Giant missions are once again under active consideration in the US as part of the 2023-2032 decadal survey, for which a flagship-class Neptune mission $\left(\right.$ Odyssey $\left.{ }^{4}\right)$ has been studied, and other concepts are likely to be considered. Furthermore, a Discovery-class Triton flyby mission (Trident, [13]) was proposed and studied in 2019-20, but unfortunately was not selected. These missions would be firmly within ESA's Voyage 2050 timeline and, provided that inter-agency collaboration supports close alignment of national and international mission opportunities, an Ice Giant partnership mission remains viable for the coming decade.

\section{Science goals of ice giant exploration}

Fletcher et al. [1] reviewed the science themes for the exploration of Ice Giant systems, which are briefly summarised here. For more thorough insights into each theme, readers are referred to two new special collections of review papers: one on Ice Giant in situ exploration in Space Science Reviews, ${ }^{5}$ and one on future exploration of Ice Giant systems in Philosophical Transactions of the Royal Society A. ${ }^{6}$ Ice Giant exploration addresses multi-disciplinary science questions, spanning comparative planetology, (exo)planetary astronomy, and heliophysics, and can be summarised via the following key themes:

1. Planetary Origins: What does the origin, interior, and divergent evolution of Uranus and Neptune reveal about the process of planetary formation, both in our Solar System and around other stars? The formation timescale of Uranus and Neptune poses a critical test of our understanding of planet formation [14], and of the architecture of our Solar System. Their bulk internal composition (i.e., the fraction of rock, ices, and gases) and the differentiation with depth (i.e.,

\footnotetext{
${ }^{2}$ https://www.nap.edu/catalog/13117/vision-and-voyages-for-planetary-science-in-the-decade-2013-2022

${ }^{3} \mathrm{http} / / /$ sci.esa.int/future-missions-department/61307-cdf-study-report-ice-giants/

${ }^{4}$ https://www.hou.usra.edu/meetings/exoplanets2020/presentations/Rymer.pdf

${ }^{5}$ https://link.springer.com/journal/11214/topicalCollection/AC_4a9035ab54970e9a8bd101f2782972b4

${ }^{6}$ http://rsta.royalsocietypublishing.org/content/378/2187
} 
molecular weight gradients, phase transitions to form global water oceans and icy mantles) are poorly understood. Indeed, the properties of the hidden interiors of Uranus and Neptune may hold the secrets to the origin and evolution of these intermediate-sized worlds, as a common outcome [15] of the planet formation process $[12,16,17]$. The Ice Giants may host exotic states of matter not found elsewhere, such as partially dissociated deep-water oceans [18] and superionic water ice mantles [19]. A detailed comparison of Uranus and Neptune may reveal the role of giant impacts [20] in shaping planetary environments. New measurements of the internal structure and rotation (via detailed mapping of gravity and magnetic fields, and possibly via helio-seismological techniques), in addition to determinations of the planetary temperatures and composition below the visible atmospheric layers via in situ probes and remote sensing, will shed new light on the origins of these two worlds.

2. Atmospheres: What processes shape the atmospheres of intermediate-sized worlds, in comparison to Gas Giants like Jupiter, or terrestrial planets like Earth? And why do atmospheric phenomena differ between Uranus and Neptune? Uranus and Neptune provide two intriguing endmembers for the Ice Giant class, with Neptune as the archetype for a seasonal Ice Giant, whereas Uranus' extreme tilt renders its seasons unique in our Solar System. Both worlds exhibit planetary bands of temperatures, winds, clouds, and composition [21] that are different from the larger Gas Giants due to the smaller planetary radii, slower rotation, and differing balance of internal energy versus solar heating [22]. The depth of the zonal flows may reveal how the atmosphere is coupled to the water-rich interior [23]. Weather causes the appearance of these worlds to change with time, and the bands are punctuated by small-scale storms and larger dark vortices that evolve and migrate over short timescales [24]. Their hydrogen-helium atmospheres are highly enriched in volatiles like methane and hydrogen sulphide that show strong equator-to-pole gradients [25, 26]. Their middle and upper atmospheres are both much hotter than can be explained by weak solar heating alone, and their stratospheres comprise a rich mix of hydrocarbons resulting from methane photochemistry [27]. Global multi-wavelength remote sensing from an orbiter, coupled with in situ measurements from an entry probe, would provide a transformative understanding of the meteorology, chemistry, and climate of atmospheres on intermediate-sized and volatile-enriched worlds, as well as the unexplored regime of weak sunlight, frigid temperatures, and extreme seasons.

3. Magnetospheres: What can we learn about astrophysical plasma processes by studying the highly-asymmetric and fast-rotating Ice Giant magnetospheres? The magnetospheres of the Ice Giants display substantial misalignments between the magnetic axes and planetary rotation axes, creating unique time-variable interactions with the external solar wind. The geometry is more complex at Uranus with the planet's high obliquity, creating a unique configuration with a strong helical structure near solstice, allowing us to test our understanding of planetary magnetospheric dynamics and evolution to the extremes [28]. The study of these unique plasma physics laboratories would 
allow us to test a variety of astrophysical plasma processes that cannot be resolved under the conditions that prevail at the terrestrial planets or at the Gas Giants, but which could be relevant to extrasolar systems [29]. A synergy between close-proximity remote sensing (e.g., of auroras or ionospheric radio emissions), and in situ magnetic field and plasma measurements at the Ice Giants would redefine the state-of-the-art.

4. Ocean Worlds: What can Uranus' natural satellite system and the captured Kuiper Belt Object Triton reveal about the drivers of active geology, subsurface oceans, and habitability in the outer Solar System? Exploring the geological and surface composition diversity [30, 31] of the five largest moons of Uranus (Miranda, Ariel, Umbriel, Titania, Oberon) would reveal new insights into the formation and continued evolution (e.g., tidal interactions, internal melting, and the potential for subsurface water oceans [32]) of primordial satellite systems, in contrast with the Saturnian moons of similar size. Only the southern hemispheres of these moons have ever been seen, due to the southern-summer season on Uranus in 1986. Neptune's large moon Triton is a captured KBO, with its own seasonal atmosphere, active geology, and plumes of nitrogen gas and dust [33], providing an intriguing connection between a future Neptune mission and the recent New Horizons exploration of Pluto [34]. Exploring Uranus' and Neptune's natural satellites, as well as the captured moon Triton, will reveal how ocean worlds form and remain active, adding new insights into the extent of the habitable zone in the Solar System.

5. Rings: What processes shape the narrow, dusty rings of Ice Giants, why do they differ from the extensive rings of Saturn, and what can they teach us about the gravitational interactions shaping planetary discs in all their guises? Both Ice Giants possess ring systems with narrow and dense ringlets accompanied by broader dusty components $[35,36]$. The composition and gravitational relationships between the rings and accompanying satellites will provide an intriguing comparison to the more extensive, brighter, and more massive rings of Saturn, and the satellites may also provide sources and sinks for some of the ring material [37]. An Ice Giant mission must explore the origin, age, evolution, and gravitational relationships within the rings as the next-best example of planetary rings after Saturn.

In addition to the Ice-Giant specific themes identified above, the White Paper [1] identified two cross-cutting interdisciplinary themes that reach beyond traditional planetary science:

- Heliophysics: How does the solar wind affect planetary environments in the realm of the Ice Giants? Exploration of an oblique rotator, and its complex interaction with the solar wind, can help inform a universal theoretical model of magnetospheres. Furthermore, the long cruise of a spacecraft beyond Saturn's orbit (10 AU) will permit extensive studies of space weather and the evolving solar structures travelling within the solar wind, in a realm that is largely unexplored [38]. The potential discoveries of Ice Giant dynamo generation and their variability stand to open new chapters in comparative planetary 
magnetospheres. For all these reasons, an Ice Giant mission is highly desirable for the Heliophysics community ${ }^{7}$.

- Exoplanets: What can the interiors, atmospheres, and magnetospheres of Uranus and Neptune teach us about one of the most common classifications (by planetary size) of exoplanets? The Ice Giants are the 'missing links' in our understanding of the origins and environments in our Solar System, and yet sub-Neptunes (1.9-3.1 $\mathrm{R}_{E}$ ), which are slightly smaller than Uranus (4.0 $\mathrm{R}_{E}$ ) and Neptune (3.8 $\mathrm{R}_{E}$ ), appear to dominate the exoplanetary census [15, 39]. The unique Ice Giant regimes of finely-tuned planet formation [14], atmospheric dynamics, bulk composition and chemistry, cloud and haze formation, and magnetospheric interactions with the stellar wind (and resulting radio emissions, [29]), will all be of interest and importance to the rapidly-expanding field of exoplanetary characterisation in the era of observatories such as JWST, TESS, the Roman Space Telescope, and Ariel.

The themes described above, and detailed in [1], demonstrate that an Ice Giant System mission would engage a wide community, drawing expertise from a vast range of disciplines within planetary science, from surface geology to planetary interiors; from meteorology to ionospheric physics; from plasma scientists to heliophysicists. But this challenge is also interdisciplinary in nature, engaging those studying potentially similar objects beyond our Solar System, by revealing the properties of this underexplored class of planetary objects. An Ice Giant System mission also draws in the small-bodies community investigating objects throughout the Outer Solar System, from Centaurs (which could be visited en route), to TNOs, to Kuiper Belt Objects, and contrasting these with the natural satellites of Uranus and Neptune.

\section{Summary: Future missions}

An ambitious, paradigm-shifting international mission to the Ice Giants has long been recognised as the next natural step in our exploration of the Outer Solar System. Following the legacy of the Cassini-Huygens exploration of the Saturn system, such a mission must combine orbital exploration of the planet, rings, satellites, and extensive magnetosphere, alongside in situ atmospheric entry probe(s) and potentially landed elements on the icy moons. A comprehensive and diverse instrument suite will enable the tremendous interdisciplinary science opportunities described in the previous section. As described in the White Paper by Fletcher et al. [1], several challenges must be overcome: the co-alignment of agency budgets and science planning to allow for international partnership missions; the strategy for dealing with long-duration missions (both technology and people) spanning multiple decades; the generation of electrical power via radioisotope decay [40]; the capabilities to return telemetry over vast distances via upgraded ground stations; and the challenge in delivering as much payload to orbit in as short a time as possible.

\footnotetext{
${ }^{7}$ https://www.nap.edu/catalog/13060/solar-and-space-physics-a-science-for-a-technological-society
} 
The use of Jupiter gravity assists (JGA), though not essential (particularly with new heavy-lift capabilities enabling direct trajectories), can shorten interplanetary transfers and enhance deliverable payload mass. However, windows of JGA opportunities only occur once every 12-13 years (the synodic periods of Uranus and Neptune with Jupiter), and the recent NASA-ESA joint study described by Hofstadter et al. [10] highlighted optimal launches to Neptune in 2029-30, and a wider window for Uranus in the early 2030s. These would have missions arriving in the 2040s, as Uranus approaches northern autumnal equinox (2050) and Neptune reaches northern spring equinox (2046) [1]. An Ice Giant launch, cruise, and scientific phase could all be encompassed within ESA's Voyage 2050 programme as a cornerstone mission, redefining planetary science for a generation. The potential for discovery is vast, and we urge our national and international space agencies to take up this challenge.

Acknowledgements Fletcher was supported by a Royal Society Research Fellowship and European Research Council Consolidator Grant (under the European Union's Horizon 2020 research and innovation programme, grant agreement No 723890) at the University of Leicester. Mousis acknowledges support from CNES. Hueso was supported by the Spanish MICINN project PID2019-109467GB-I00 (MICINN/FEDER, UE) and by Grupos Gobierno Vasco IT1366-19 from Gobierno Vasco. UK authors acknowledge support from the Science and Technology Facilities Council (STFC). Tortora was supported by ASI (Italian Space Agency) under grant agreement 2020-13-HH.0 We are grateful to the members of ESA's Voyage 2050 Senior Committee for allowing us to present this review at the Voyage 2050 workshop in Madrid in October 2019.

Open Access This article is licensed under a Creative Commons Attribution 4.0 International License, which permits use, sharing, adaptation, distribution and reproduction in any medium or format, as long as you give appropriate credit to the original author(s) and the source, provide a link to the Creative Commons licence, and indicate if changes were made. The images or other third party material in this article are included in the article's Creative Commons licence, unless indicated otherwise in a credit line to the material. If material is not included in the article's Creative Commons licence and your intended use is not permitted by statutory regulation or exceeds the permitted use, you will need to obtain permission directly from the copyright holder. To view a copy of this licence, visit http://creativecommons.org/licenses/by/4. $0 /$.

\section{References}

1. Fletcher, L.N., Helled, R., Roussos, E., Jones, G., Charnoz, S., André, N., Andrews, D., Bannister, M., Bunce, E., Cavalié, T., Ferri, F., Fortney, J., Grassi, D., Griton, L., Hartogh, P., Hueso, R., Kaspi, Y., Lamy, L., Masters, A.., Melin, H.., Moses, J., Mousis, O., Nettleman, N., Plainaki, C., Schmidt, J., Simon, A., Tobie, G., Tortora, P., Tosi, F., Turrini, D.: Plan. \& Space Sci. 191, 105030 (2020). https://doi.org/10.1016/j.pss.2020.105030

2. Stone, E.C., Miner, E.D.: Science 233(4759), 39-43 (1986). https://doi.org/10.1126/science.233. 4759.39

3. Stone, E.C., Miner, E.D.: Science 246(4936), 1417-1421 (1989). https://doi.org/10.1126/sci ence.246.4936.1417

4. Smith, B.A., Soderblom, L.A., Beebe, R., Bliss, D., Boyce, J.M., Brahic, A., Briggs, G.A., Brown, R.H., Collins, S.A., Cook, A.F., Croft, S.K., Cuzzi, J.N., Danielson, G.E., Davies, M.E., Dowling, T.E., Godfrey, D., Hansen, C.J., Harris, C., Hunt, G.E., Ingersoll, A.P., Johnson, T.V., Krauss, R.J., Masursky, H., Morrison, D., Owen, T., Plescia, J.B., Pollack, J.B., Porco, C.C., Rages, K., Sagan, C., Shoemaker, E.M., Sromovsky, L.A., Stoker, C., Strom, R.G., Suomi, V.E., Synnott, 
S.P., Terrile, R.J., Thomas, P., Thompson, W.R., Veverka, J.: Science 233(4759), $43-64$ (1986). https://doi.org/10.1126/science.233.4759.43

5. Smith, B.A., Soderblom, L.A., Banfield, D., Barnet, C., Basilevsky, A.T., Beebe, R.F., Bollinger, K., Boyce, J.M., Brahic, A., Briggs, G.A., Brown, R.H., Chyba, C., Collins, S.A., Colvin, T., Cook, A.F., Crisp, D., Croft, S.K., Cruikshank, D., Cuzzi, J.N., Danielson, G.E., Davies, M.E., De Jong, E., Dones, L., Godfrey, D., Goguen, J., Grenier, I., Haemmerle, V.R., Hammel, H., Hansen, C.J., Helfenstein, C.P., Howell, C., Hunt, G.E., Ingersoll, A.P., Johnson, T.V., Kargel, J., Kirk, R., Kuehn, D.I., Limaye, S., Masursky, H., McEwen, A., Morrison, D., Owen, T., Owen, W., Pollack, J.B., Porco, C.C., Rages, K., Rogers, P., Rudy, D., Sagan, C., Schwartz, J., Shoemaker, E.M., Showalter, M., Sicardy, B., Simonelli, D., Spencer, J., Sromovsky, L.A., Stoker, C., Strom, R.G., Suomi, V.E., Synott, S.P., Terrile, R.J., Thomas, P., Thompson, W.R., Verbiscer, A., Veverka, J.: Science 246, 1422-1449 (1989). https://doi.org/10.1126/science.246.4936.1422

6. Arridge, C.S., Agnor, C.B., André, N., Baines, K.H., Fletcher, L.N., Gautier, D., Hofstadter, M.D., Jones, G.H., Lamy, L., Langevin, Y., Mousis, O., Nettelmann, N., Russell, C.T., Stallard, T., Tiscareno, M.S., Tobie, G., Bacon, A., Chaloner, C., Guest, M., Kemble, S., Peacocke, L., Achilleos, N., Andert, T.P., Banfield, D., Barabash, S., Barthelemy, M., Bertucci, C., Brandt, P., Cecconi, B., Chakrabarti, S., Cheng, A.F., Christensen, U., Christou, A., Coates, A.J., Collinson, G., Cooper, J.F., Courtin, R., Dougherty, M.K., Ebert, R.W., Entradas, M., Fazakerley, A.N., Fortney, J.J., Galand, M.., Gustin, J., Hedman, M., Helled, R., Henri, P., Hess, S., Holme, R.., Karatekin, O., Krupp, N., Leisner, J., Martin-Torres, J.,, Masters, A., Melin, H., Miller, S., Müller-Wodarg, I., Noyelles, B., Paranicas, C., de Pater, I., Pätzold, M., Prangé, R., Quémerais, E., Roussos, E., Rymer, A.M., Sánchez-Lavega, A., Saur, J., Sayanagi, K.M., Schenk, P., Schubert, G.., Sergis, N., Sohl, F., Sittler, E.C., Teanby, N.A., Tellmann, S., Turtle, E.P., Vinatier, S., Wahlund, J.-E., Zarka, P.: Exp. Astron. 33, 753-791 (2012). https://doi.org/10.1007/s10686-011-9251-4

7. Arridge, C.S., Achilleos, N., Agarwal, J., Agnor, C.B., Ambrosi, R., André, N., Badman, S.V., Baines, K., Banfield, D., Barthélémy, M., Bisi, M.M., Blum, J., Bocanegra-Bahamon, T., Bonfond, B., Bracken, C., Brandt, P., Briand, C., Briois, C., Brooks, S., Castillo-Rogez, J., Cavalié, T., Christophe, B., Coates, A.J., Collinson, G., Cooper, J.F., Costa-Sitja, M., Courtin, R., Daglis, I.A., de Pater, I., Desai, M., Dirkx, D., Dougherty, M.K., Ebert, R.W., Filacchione, G., Fletcher, L.N., Fortney, J., Gerth, I., Grassi, D., Grodent, D., Grün, E., Gustin, J., Hedman, M., Helled, R., Henri, P., Hess, S., Hillier, J.K., Hofstadter, M.H., Holme, R., Horanyi, M., Hospodarsky, G., Hsu, S., Irwin, P., Jackman, C.M., Karatekin, O., Kempf, S., Khalisi, E., Konstantinidis, K., Krüger, H., Kurth, W.S., Labrianidis, C., Lainey, V., Lamy, L.L., Laneuville, M., Lucchesi, D., Luntzer, A., MacArthur, J., Maier, A., Masters, A., McKenna-Lawlor, S., Melin, H., Milillo, A., Moragas-Klostermeyer, G., Morschhauser, A., Moses, J.I., Mousis, O., Nettelmann, N., Neubauer, F.M., Nordheim, T., Noyelles, B., Orton, G.S., Owens, M., Peron, R., Plainaki, C., Postberg, F., Rambaux, N., Retherford, K., Reynaud, S., Roussos, E., Russell, C.T., Rymer, A.M., Sallantin, R., Sánchez-Lavega, A., Santolik, O., Saur, J., Sayanagi, K.M., Schenk, P., Schubert, J., Sergis, N., Sittler, E.C., Smith, A., Spahn, F., Srama, R., Stallard, T., Sterken, V., Sternovsky, Z., Tiscareno, M., Tobie, G., Tosi, F., Trieloff, M., Turrini, D., Turtle, E.P., Vinatier, S., Wilson, R., Zarka, P.: Plan. \& Space Sci. 104, 122-140 (2014). https://doi.org/10.1016/j.pss.2014.08.009

8. Masters, A., Achilleos, N., Agnor, C.B., Campagnola, S., Charnoz, S., Christophe, B., Coates, A.J., Fletcher, L.N., Jones, G.H., Lamy, L., Marzari, F., Nettelmann, N., Ruiz, J., Ambrosi, R., Andre, N., Bhardwaj, A., Fortney, J.J., Hansen, C.J., Helled, R., Moragas-Klostermeyer, G., Orton, G., Ray, L., Reynaud, S., Sergis, N., Srama, R., Volwerk, M.: Plan. Space Sci. 104, 108-121 (2014). https://doi.org/10.1016/j.pss.2014.05.008

9. Turrini, D., Politi, R., Peron, R., Grassi, D., Plainaki, C., Barbieri, M., Lucchesi, D.M., Magni, G., Altieri, F., Cottini, V., Gorius, N., Gaulme, P., Schmider, F.-X., Adriani, A., Piccioni, G.: Plan. Space Sci. 104, 93-107 (2014). https://doi.org/10.1016/j.pss.2014.09.005

10. Hofstadter, M., Simon, A., Atreya, S., Banfield, D., Fortney, J.J., Hayes, A., Hedman, M., Hospodarsky, G., Mandt, K., Masters, A., Showalter, M., Soderlund, K.M., Turrini, D., Turtle, E., Reh, K., Elliott, J., Arora, N., Petropoulos, A.: Plan. Space Sci. 177, 104680 (2019). https://doi.org/10.1016/j.pss.2019.06.004

11. Simon, A.A., Fletcher, L.N., Arridge, C., Atkinson, D., Coustenis, A., Ferri, F., Hofstadter, M., Masters, A., Mousis, O., Reh, K., Turrini, D., Witasse, O.: Space Sci. Rev. 216(1), 17 (2020). https://doi.org/10.1007/s11214-020-0639-1 
12. Mousis, O., Atkinson, D.H., Cavalié, T., Fletcher, L.N., Amato, M.J., Aslam, S., Ferri, F., Renard, J.-B., Spilker, T., Venkatapathy, E., Wurz, P., Aplin, K., Coustenis, A., Deleuil, M., Dobrijevic, M., Fouchet, T., Guillot, T., Hartogh, P., Hewagama, T., Hofstadter, M.D., Hue, V., Hueso, R., Lebreton, J.-P., Lellouch, E., Moses, J., Orton, G.S., Pearl, J.C., Sánchez-Lavega, A., Simon, A., Venot, O., Waite, J.H., Achterberg, R.K., Atreya, S., Billebaud, F., Blanc, M., Borget, F., Brugger, B., Charnoz, S., Chiavassa, T., Cottini, V., d'Hendecourt, L., Danger, G., Encrenaz, T., Gorius, N.J.P., Jorda, L., Marty, B., Moreno, R., Morse, A., Nixon, C., Reh, K., Ronnet, T., Schmider, F.X., Sheridan, S., Sotin, C., Vernazza, P., Villanueva, G.L.: Plan. Space Sci. 155, $12-40$ (2018). https://doi.org/10.1016/j.pss.2017.10.005

13. Prockter, L.M., Mitchell, K.L., Howett, C.J.A., Smythe, W.D., Sutin, B.M., Bearden, D.A., Frazier, W.E.: In: Lunar and Planetary Science Conference, Lunar and Planetary Science Conference, pp. 3188 (2019)

14. Venturini, J., Helled, R.: Astrophys. J. 848(2), 95 (2017). https://doi.org/10.3847/1538-4357/aa8cd0

15. Fulton, B.J., Petigura, E.A.: Astron. J. 156(6), 264 (2018). https://doi.org/10.3847/1538-3881/aae828

16. Atreya, S.K., Hofstadter, M.H., In, J.H., Mousis, O., Reh, K., Wong, M.H.: Space Sci. Rev. 216(1), 18 (2020). https://doi.org/10.1007/s11214-020-0640-8

17. Helled, R., Nettelmann, N., Guillot, T.: Space Sci. Rev. 216(3), 38 (2020). https://doi.org/10.1007/ s11214-020-00660-3

18. Redmer, R., Mattsson, T.R., Nettelmann, N., French, M.: Icarus 211(1), 798-803 (2011). https://doi.org/10.1016/j.icarus.2010.08.008

19. Millot, M., Hamel, S., Rygg, J.R., Celliers, P.M., Collins, G.W., Coppari, F., Fratanduono, D.E., Jeanloz, R., Swift, D.C., Eggert, J.H.: Nat. Phys. 14(3), 297-302 (2018). https://doi.org/10.1038/s41567-017-0017-4

20. Kegerreis, J.A., Eke, V.R., Gonnet, P., Korycansky, D.G., Massey, R.J., Schaller, M., Teodoro, L.F.A.: Mon. Not. R. Astron. Soc. 487(4), 5029-5040 (2019). https://doi.org/10.1093/mnras/stz1606

21. Fletcher, L.N., de Pater, I., Orton, G.S., Hofstadter, M.D., Irwin, P.G.J., Roman, M.T., Toledo, D.: Space Sci. Rev. 216(2), 21 (February 2020). https://doi.org/10.1007/s11214-020-00646-1

22. Pearl, J.C., Conrath, B.J.: J. Geophys. Res. 96, 18921 (1991)

23. Kaspi, Y., Showman, A.P., Hubbard, W.B., Aharonson, O., Helled, R.: Nature 497, 344-347 (2013). https://doi.org/10.1038/nature12131

24. Hueso, R., Sánchez-Lavega, A.: Space Sci. Rev. 215(8), 52 (2019). https://doi.org/10.1007/s11214019-0618-6

25. Karkoschka, E., Tomasko, M.: Icarus 202, 287-309 (2009). https://doi.org/10.1016/j.icarus.2009. 02.010

26. Tollefson, J., de Pater, I., Luszcz-Cook, S., DeBoer, D.: Astrophys. J. 157(6), 251 (2019). https://doi. org/10.3847/1538-3881/ab1fdf, arXiv: 1905.03384

27. Moses, J.I., Fletcher, L.N., Greathouse, T.K., Orton, G.S., Hue, V.: Icarus 307, 124-145 (2018). https://doi.org/10.1016/j.icarus.2018.02.004

28. Arridge, C.S., Paty, C.: In: Maggiolo, R., André, N., Hasegawa, H., Welling, D. (eds.) Magnetospheres in the Solar System, Space Physics and Aeronomy. Wiley (2020)

29. Rymer, A., Mandt, K., Hurley, D., Lisse, C., Izenberg, N., Smith, H.T., Westlake, J., Bunce, E., Arridge, C., Masters, A., Hofstadter, M., Simon, A., Brand t, P., Clark, G., Cohen, I., Allen, R., Vine, S., Hansen, K., Hospodarsky, G., Kurth, W., Romani, P., Lamy, L., Zarka, P., Cao, H., Paty, C., Hedman, M., Roussos, E., Cruikshank, D., Farrell, W., Fieseler, P., Coates, A., Yelle, R., Parkinson, C., Militzer, B., Grodent, D., Kollmann, P., McNutt, R., André, N., Strange, N., Barnes, J., Dones, L., Denk, T., Rathbun, J., Lunine, J., Desai, R., Cochrane, C., Sayanagi, K.M., Postberg, F., Ebert2, R., Hill, T., Mueller-Wodarg, I., Regoli, L., Pontius, D., Stanley, S., Greathouse, T., Saur, J., Marouf, E., Bergman, J., Higgins, C., Johnson, R., Thomsen, M., Soderlund, K., Jia, X., Wilson, R., England er, J., Burch, J., Nordheim, T., Grava, C., Baines, K., Quick, L., Russell, C., Cravens, T., Cecconi, B., Aslam, S., Bray, V., Garcia-Sage, K., Richardson, J., Clark, J., Hsu, S., Achterberg, R., Sergis, N., Paganelli, F., Kempf, S., Orton, G., Portyankina, G., Jones, G., Economou, T., Livengood, T., Krimigis, S., Szalay, J., Jackman, C., Valek, P., Lecacheux, A., Colwell, J., Jasinski, J., Tosi, F., Sulaiman, A., Galand, M., Kotova, A., Khurana, K., Kivelson, M., Strobel, D., Radiota, A., Estrada, P., Livi, S., Azari, A., Yates, J., Allegrini, F., Vogt, M., Felici, M., Luhmann, J., Filacchione, G., Moore, L.: Bull. Am. Astron. Soc. 51(3), 176 (2019)

30. Grundy, W.M., Young, L.A., Spencer, J.R., Johnson, R.E., Young, E.F., Buie, M.W.: Icarus 184(2), 543-555 (2006). https://doi.org/10.1016/j.icarus.2006.04.016 
31. Cartwright, R.J., Emery, J.P., Pinilla-Alonso, N., Lucas, M.P., Rivkin, A.S., Trilling, D.E.: Icarus 314, 210-231 (2018). https://doi.org/10.1016/j.icarus.2018.06.004

32. Hussmann, H., Sohl, F., Spohn, T.: Icarus 185(1), 258-273 (2006). https://doi.org/10.1016/j.icarus. 2006.06.005

33. Soderblom, L.A., Kieffer, S.W., Becker, T.L., Brown, R.H., Cook, I.I., Hansen, C.J., Johnson, T.V., Kirk, R.L., Shoemaker, E.M.: Science 250(4979), 410-415 (1990). https://doi.org/10.1126/science. 250.4979.410

34. Grundy, W.M., Binzel, R.P., Buratti, B.J., Cook, J.C., Cruikshank, D.P., Dalle Ore, C.M., Earle, A.M., Ennico, K., Howett, C.J.A., Lunsford, A.W., Olkin, C.B., Parker, A.H., Philippe, S., Protopapa, S., Quirico, E., Reuter, D.C., Schmitt, B., Singer, K.N., Verbiscer, A.J., Beyer, R.A., Buie, M.W., Cheng, A.F., Jennings, D.E., Linscott, I.R., Parker, J.W., Schenk, P.M., Spencer, J.R., Stansberry, J.A., Stern, S.A., Throop, H.B., Tsang, C.C.C., Weaver, H.A., Weigle, G.E., Young, L.A.: Science 351(6279), aad9189 (2016). https://doi.org/10.1126/science.aad9189

35. Nicholson, P.D., Pater, I.D., French, R.G., Showalter, M.R.: In: Tiscareno, M.S., Murray, C.D.E. (eds.) Planetary Ring Systems: Properties, Structure, and Evolution, Cambridge Planetary Science, pp. 93111. Cambridge University Press (2018)

36. de Pater, I., Renner, S., Showalter, M.R., Sicardy, B.: In: Tiscareno, M.S., Murray, C.D.E. (eds.) Planetary Ring Systems: Properties, Structure, and Evolution, Cambridge Planetary Science, pp. 112124. Cambridge University Press (2018)

37. de Pater, I., Hammel, H.B., Gibbard, S.G., Showalter, M.R.: Science 312(5770), 92-94 (2006). https://doi.org/10.1126/science. 1125110

38. Witasse, O., Sánchez-Cano, B., Mays, M.L., Kajdič, P., Opgenoorth, H., Elliott, H.A., Richardson, I.G., Zouganelis, I., Zender, J., Wimmer-Schweingruber, R.F., Turc, L., Taylor, M.G.G.T., Roussos, E., Rouillard, A., Richter, I., Richardson, J.D., Ramstad, R., Provan, G., Posner, A., Plaut, J.J., Odstrcil, D., Nilsson, H., Niemenen, P., Milan, S.E., Mandt, K., Lohf, H., Lester, M., Lebreton, J.P., Kuulkers, E., Krupp, N., Koenders, C., James, M.K., Intzekara, D., Holmstrom, M., Hassler, D.M., Hall, B.E.S., Guo, J., Goldstein, R., Goetz, C., Glassmeier, K.H., Génot, V., Evans, H., Espley, J., Edberg, N.J.T., Dougherty, M., Cowley, S.W.H., Burch, J., Behar, E., Barabash, S., Andrews, D.J., Altobelli, N.: J. Geophys. Res. (Space Phys.) 122(8), 7865-7890 (2017). https://doi.org/10.1002/2017JA023884

39. Wakeford, H.R., Dalba, P.A.: Philos. Trans. R. Soc. Lond. Ser. A 378(2187), 20200054 (2020). https://doi.org/10.1098/rsta.2020.0054

40. Ambrosi, R.M., Williams, H., Watkinson, E.J., Barco, A., Mesalam, R., Crawford, T., Bicknell, C., Samara-Ratna, P., Vernon, D., Bannister, N., Ross, D., Sykes, J., Perkinson, M.-C., Burgess, C., Stroud, C., Gibson, S., Godfrey, A., Slater, R.G., Reece, M.J., Chen, K., Simpson, K., Tuley, R., Sarsfield, M., Tinsley, T.P., Stephenson, K., Freis, D., Vigier, J.-F., Konings, R.J.M., Fongarland, C., Libessart, M., Merrifield, J., Kramer, D.P., Byrne, J., Foxcroft, B.: Space Sci. Rev. 215(8), 55 (2019). https://doi.org/10.1007/s11214-019-0623-9

Publisher's note Springer Nature remains neutral with regard to jurisdictional claims in published maps and institutional affiliations. 


\section{Affiliations}

Leigh N. Fletcher ${ }^{1}(1)$. Ravit Helled ${ }^{2}$. Elias Roussos ${ }^{3}$. Geraint Jones ${ }^{4}$. Sébastien Charnoz ${ }^{5}$. Nicolas André ${ }^{6}$. David Andrews ${ }^{7} \cdot$ Michele Bannister $^{8}$. Emma Bunce ${ }^{1}$. Thibault Cavalié ${ }^{9,10}$. Francesca Ferri ${ }^{11}$. Jonathan Fortney ${ }^{12}$. Davide Grassi ${ }^{13}$. Léa Griton ${ }^{14}$. Paul Hartogh ${ }^{3}$. Ricardo Hueso ${ }^{15}$. Yohai Kaspi ${ }^{16}$. Laurent Lamy ${ }^{17}$. Adam Masters ${ }^{18}$. Henrik Melin ${ }^{1}$. Julianne Moses ${ }^{19}$. Oliver Mousis ${ }^{20}$. Nadine Nettleman ${ }^{21}$. Christina Plainaki ${ }^{22}$. Jürgen Schmidtt ${ }^{23}$. Amy Simon ${ }^{24}$. Gabriel Tobie ${ }^{25}$ • Paolo Tortora ${ }^{26}$. Federico Tosi $^{13} \cdot$ Diego Turrini $^{13}$

1 School of Physics and Astronomy, University of Leicester, University Road, Leicester, LE1 7RH, UK

2 University of Zurich, Zurich, Switzerland

3 Max Planck Institute for Solar System Research, Göttingen, Germany

4 Mullard Space Science Laboratory, Dorking, UK

5 Institut de Physique du Globe de Paris, Paris, France

6 Institut de Recherche en Astrophysique et Planétologie, Toulouse, France

7 Swedish Institute of Space Physics, Kiruna, Sweden

8 University of Canterbury, Canterbury, New Zealand

9 Laboratoire d'Astrophysique de Bordeaux, University of Bordeaux, CNRS, Pessac, France

10 LESIA, Observatoire de Paris, Université PSL, CNRS, Sorbonne Université, Univ. Paris Diderot, Sorbonne Paris Cité, Meudon, France

Università degli Studi di Padova, Padova, Italy

12 University of Santa-Cruz, Santa-Cruz, USA

13 Istituto Nazionale di AstroFisica - Istituto di Astrofisica e Planetologia Spaziali (INAF-IAPS), Rome, Italy

14 Institut de Recherche en Astrophysique et Planétologie, CNRS, Toulouse, France

15 Escuela de Ingeniería de Bilbao, UPV/EHU, Bilbao, Spain

16 Weizmann Institute of Science, Rehobot, Israel

17 Observatoire de Paris, Paris, France

18 Imperial College London, London, UK

19 Space Science Institute, Colorado, USA

20 CNRS, CNES, LAM, Aix Marseille University, Marseille, France

21 University of Rostock, Rostock, Germany ASI - Italian Space Agency, Rome, Italy

23 University of Oulu, Oulu, Finland

24 NASA Goddard Space Flight Center, Greenbelt, USA

25 Université de Nantes, Nantes, France

26 Università di Bologna, Bologna, Italy 